\title{
Indications for investigation of chronic gastrointestinal symptoms
}

\author{
R M Beattie, J A Walker-Smith, S H Murch
}

\begin{abstract}
Simple routine blood tests (full blood count, platelet count, erythrocyte sedimentation rate, $C$ reactive protein, and serum albumin) may select children with chronic gastrointestinal symptoms who require endoscopic assessment and are thus an important aid in the prediction of endoscopic status. When all of the results are normal then chronic inflammatory bowel disease is an unlikely diagnosis. (Arch Dis Child 1995; 73: 354-355)
\end{abstract}

Keywords: Crohn's disease, screening, abdominal pain, $\mathrm{C}$ reactive protein.

Colonoscopy is indicated in children with chronic gastrointestinal symptoms in order to diagnose chronic inflammatory bowel diseases, polyps, and less common conditions. Many children who undergo endoscopy are, however, found to have no organic disease. Additionally in Crohn's disease, the commonest inflammatory bowel disease, diagnosis is often delayed, and despite the availability of paediatric colonoscopy the median time to diagnosis from onset of symptoms is still around 15 months. ${ }^{1}$ As the early symptoms of Crohn's disease are often non-specific, diagnosis is often dependent upon investigations, in particular contrast radiology, endoscopy, and histology. Although abdominal pain is an important symptom of Crohn's disease, it is also a common childhood complaint. The selection of whom to refer for endoscopy may therefore be difficult: use of screening blood tests to aid in patient selection is therefore likely to be of benefit.

We have audited all tertiary referrals to our unit for diagnostic colonoscopy over an 18 month period (January 1993-August 1994), and correlated the endoscopic diagnosis with the results of easily available screening investigations.

Patients, methods, and results

Academic Department of Paediatric Gastroenterology, St Bartholomew's Hospital, London R M Beattie J A Walker-Smith S H Murch

Correspondence to: Dr R M Beattie, Paediatric Department, Peterborough District Hospital, Thorpe Road, Peterborough PE3 6DA. Accepted 14 June 1995

\section{Patients, methods, and resuits}

One hundred and twenty four patients were referred for endoscopic assessment by consultant paediatricians over an 18 month period. In 93 children the diagnosis was uncertain. All patients reported two or more of the following symptoms: abdominal pain, diarrhoea, rectal bleeding, weight loss, or mouth ulceration for greater than three months. The median age of the group was 11 years (range 3 months-18 years) and median duration of symptoms seven months (range 3-60).

One patient was excluded because of short duration of symptoms (two weeks) and one because of orofacial granulomatosis. Thus 91 patients fulfilled the criteria for inclusion.

All patients underwent endoscopy and barium follow through at initial assessment. Endoscopic assessment included total colonoscopy and ileoscopy with biopsy. No patient in the study had abnormal radiology with a normal endoscopic assessment and therefore outcome was correlated with endoscopic diagnosis.

Screening variables assessed were haemoglobin concentration, platelet count, erythrocyte sedimentation rate, $C$ reactive protein, and serum albumin, arranged routinely, using standard techniques, by our haematology and biochemistry laboratories.

Diagnosis was established in all 91 children: 26 were found to have Crohn's disease, 13 ulcerative colitis, eight had polyps, and 37 were normal. Other diagnoses included tuberculosis (2), indeterminate colitis (3), and lymphoid nodular hyperplasia (2).

The results of screening investigations are shown in the table. Overall no child with Crohn's disease had fully normal screening investigations, although one child with ulcerative colitis had normal investigations despite a clear history of rectal bleeding. Two of 37 children with a normal endoscopic assessment had a raised platelet count and one was anaemic.

\section{Discussion}

Our results show that simple routine blood tests may be valuable in the selection of children with gastrointestinal symptoms who require endoscopic assessment. In particular all children with undiagnosed Crohn's disease had a $C$ reactive protein greater than $5 \mathrm{mg} / \mathrm{l}$. Normal indices do not invariably mean that endoscopy will be normal: either polyposis or ulcerative colitis may be present. However, most of these patients have rectal bleeding which should make colonoscopy mandatory. The diagnosis of Crohn's disease is unlikely if screening investigations are normal, but should be strongly considered if these indices are abnormal. The diagnosis of ulcerative colitis is unlikely if the indices are all normal and rectal bleeding is not present.

The role of blood tests in the assessment of known chronic inflammatory bowel disease is well established. ${ }^{2-4}$ Harries et al focused particularly on the platelet count as a marker of inflammatory activity in Crohn's disease. ${ }^{3} \mathrm{We}$ have found the $C$ reactive protein to be the most sensitive inflammatory indicator at diagnosis. C reactive protein reflects the systemic response to locally produced cytokines from 
Relationship between endoscopic diagnosis and routine laboratory tests in children with chronic gastrointestinal symptoms; values are \%

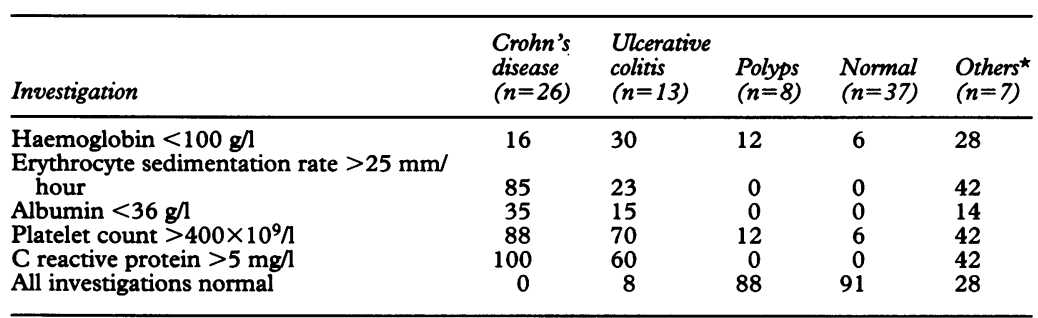

*Others include tuberculosis (2), indeterminate colitis (3), and lymphoid nodular hyperplasia (2).

the gut mucosa and is a very sensitive indicator of disease activity in Crohn's disease. ${ }^{5}$

The use of inflammatory markers to aid the approach to diagnosis has been investigated in adults. ${ }^{6}$ Shine et al reported a series of 82 patients with chronic gastrointestinal symptoms assessed by clinical history, erythrocyte sedimentation rate, $\mathrm{C}$ reactive protein, and rectal biopsy. They found that, in the group with irritable bowel syndrome/functional disorder', no patient had a raised $\mathrm{C}$ reactive protein and erythrocyte sedimentation rate. In those with Crohn's disease, indices were raised in 19/19 and in ulcerative colitis in $11 / 22$. Thus normal investigations may be of use in avoiding total colonoscopy in patients with functional abdominal pain. This is even more important in children.

Most of the children with Crohn's disease at endoscopy also had abnormalities on the barium meal and follow through (24/26).
Barium meal and follow through is a more readily available investigation. Endoscopy with biopsy, in addition to small bowel radiology, however, is worthwhile in order to assess disease extent - which may influence treatment - and to confirm the diagnosis.

We suggest that these simple tests should be performed early in all children with chronic gastrointestinal symptoms. Abnormalities suggest the need for early referral, whereas normal investigations and no history of rectal bleeding make chronic inflammatory bowel disease unlikely. This policy should lead to swifter diagnosis in children with inflammatory bowel disease and may spare many others invasive investigations.

This study was supported by the Crohn's in Childhood Research Association.

1 Beasley SW, Cameron DIS, McLain BI, Davidson PM, Stokes KB. Diagnostic delay in Crohn's disease. Paediatr Stokes KB. Diagnos

2 Campbell CA, Walker-Smith JA, Hindocha P, Adinolfi $M$ Acute phase proteins in chronic inflammatory bowel disease in childhood. $\mathcal{F}$ Pediatr Gastroenterol Nutr 1982; 1 : 193-201.

3 Harries AD, Fitszimmons E, Fifield R, Dew MJ, Rhodes J. Platelet count: a simple measure of activity in Crohn's disease. $B M \mathcal{Y}$ 1983; 286: 1476-7.

4 MacFarlane PI, Miller V, Wells F, Richards B. Laboratory assessment of disease activity in childhood Crohn's disease and ulcerative colitis. $\mathcal{F}$ Pediatr Gastroenterol Nutr 1986; 5: 93-6.

5 Murch SH, Lamkin VA, Savage MO, Walker-Smith JA, MacDonald TT. Serum concentrations of tumour necrosis factor alpha in childhood chronic inflammatory bowel disease. Gut 1991; 32: 913-7.

6 Shine B, Berghouse L, Lennard-Jones JE, Landon J. C-Reactive protein as an aid in the differential diagnosis of functional and inflammatory bowel disorders. Clin Chim Acta 1985; 148: 105-9. 